\title{
Modeling the Delayed Emission in the 2005 Mkn 501 Flare
}

Robert Wagner*

Max-Planck-Institut für Physik, Föhringer Ring 6, D-80805 München

E-mail: robert.wagner@mpp.mpg.de

\section{Włodek Bednarek}

Department of Experimental Physics, University of Łódź, ul. Pomorska 149/153, PL-90-236

Łódź, Poland

Recently, the MAGIC collaboration reported evidence for a delay in the arrival times of photons of different energies during a $\gamma$-ray flare from the blazar Markarian 501 on 2005 July 9. We describe the observed delayed high-energy emission by applying a homogeneous synchrotron self-Compton (SSC) model under the assumption that the blob containing relativistic electrons was observed in its acceleration phase. This modified SSC model predicts the appearance of a $\gamma$-ray flare first at lower energies and subsequently at higher energies. Based on the reported time delay of $\sim 240$ s between the flare observed at $\sim 190 \mathrm{GeV}$ and $2.7 \mathrm{TeV}$, we predict a delay on the order of $1 \mathrm{~h}$ if observed between $10 \mathrm{GeV}$ and $100 \mathrm{GeV}$. Such delay timescales can be tested in the future by simultaneous flare observations with the Gamma Ray Large Area Space Telescope (GLAST) and Cerenkov telescopes.

Workshop on Blazar Variability across the Electromagnetic Spectrum April 22-25 2008

Palaiseau, France

\footnotetext{
* Speaker.
} 


\section{Introduction}

Recent observations of energy-dependent, few-minute timescale $\mathrm{TeV} \gamma$-ray flares in the blazar Markarian (Mkn) 501 [1] are difficult to explain in terms of the "classical" homogeneous synchrotron self-Compton (SSC) model (e.g., [2,3]) in which the emission region is moving along the jet with a constant Lorentz factor $\sim 10-20$ [4], as inferred from the observations of superluminal motion in active galactic nuclei. Similar few-minute timescale flares have also been observed recently from PKS 2155-304 [5, 6]. However, in the case of PKS 2155-304, no energy-dependent flare evolution was found [7]. Homogeneous blob models require blobs moving with Lorentz factors significantly larger than mentioned above, see, e.g., fig. 3c in [8], or [9, 5]. In the case of the 2005 July 9 flare of Mkn 501, an arrival time delay was observed between $\gamma$-rays in the $>1.2 \mathrm{TeV}$ energy range with respect to the $0.15-0.25 \mathrm{TeV}$ energy range [1]. Two solutions for these fine emission features have been considered up to now. In the gradual-acceleration scenario, particles inside the emission region moving with constant Doppler factor need some time to be accelerated to energies that allow them to produce $\gamma$-rays with specific energies. Therefore, a $\gamma$-ray flare should first appear at lower energies and only later on at larger energies. The second scenario relates the delay between different energies to the energy-dependent speed of photons in vacuum. The effect that lower-energy photons arrive before the higher-energy ones is predicted in some models of quantum-gravity (see [10] for details).

The large Doppler factors inferred from $\mathrm{TeV} \gamma$-ray observations have to be achieved in the very inner parts of the jet. Therefore, it is natural to expect that the emission region has to undergo an initial acceleration phase. Very-Large Baseline Interferometry (VLBI) observations rather show evidence for decelerating radio-emitting blobs (e.g., [11] for Mkn 501). However, the very inner regions where we assume the initial acceleration to take place, could not be investigated yet with VLBI.

Here we show that a delayed emission of the higher-energy $\gamma$-rays can be naturally explained by assuming that the blob accelerates significantly during its propagation along the jet, producing more energetic $\gamma$-rays when the Lorentz factor of the blob is larger. We argue that for the first time $\gamma$-ray emission in BL Lac objects has been captured in the initial acceleration phase of the blob. This model is presented in detail also in [12].

\section{Accelerating blob model}

We consider a blob of relativistic electrons that moves along the jet with a Lorentz factor $\gamma_{b}$ at any specific moment. We assume a "classical" SSC model, in which electrons are accelerated inside the blob by shock acceleration, with an acceleration efficiency proportional to the magnetic field strength at the location of the blob. Due to the presence of magnetic fields, the electrons inside the blob lose energy by synchrotron emission. This soft radiation is upscattered to $\gamma$-ray energies by the same population of electrons. In terms of this classical picture, $\mathrm{X}$-ray and $\mathrm{TeV} \gamma$-ray flares are naturally explained. However, the observation of a time delay between $\gamma$-rays of different energies belonging to the same flare cannot be easily understood in such a simple scenario. Therefore, we modify the homogeneous SSC model by assuming that the blob has been captured during its acceleration phase in the inner part of the jet. Let us consider the blob acceleration between its 
Lorentz factors $\gamma_{b}^{\min }$ and $\gamma_{b}^{\max }$. We assume that the magnetic field strength in the jet (and also in the blob) drops proportionally to the distance from the base of the jet, $B \propto X^{-1}$, which is a good approximation in the case of a conical jet with a fixed opening angle at some distance to the base of the jet. In our model, we discuss a quasi-spherical blob with a radius $R_{\mathrm{b}} \propto X$, i.e., proportional to its distance from the base of the jet. This is expected for a conical jet, whose borders limit the expansion of the blob.

We propose that a delay observed in a flare between different energy ranges is due to the fact that the lower-energy part of the flare is produced when the blob is closer to the base of the jet (moving with a lower Lorentz factor), while the higher-energy part is produced at larger distances from the jet base, at which the blob has accelerated to a higher Lorentz factor.

We now derive a description of the acceleration of the blob along the jet. Observations show that the TeV $\gamma$-ray luminosities found for Mkn 501 during the 2005 July 9 flare in the abovementioned two energy ranges are comparable [1]. The observed $\gamma$-ray luminosity is related to the luminosity in the blob frame by

$$
\dot{P}_{\mathrm{obs}}^{\mathrm{IC}} \propto \dot{P}_{\mathrm{b}}^{\mathrm{IC}} D_{\mathrm{b}}^{2}
$$

where $D_{\mathrm{b}}$ is the Doppler factor of the blob. In the simplest possible case, in which the observer is located on the axis of the jet, the blob Doppler factor is related to its Lorentz factor by $D_{\mathrm{b}}=$ $\left[\gamma_{\mathrm{b}}\left(1-\beta_{\mathrm{b}} \cos \theta\right)\right]^{-1} \simeq 2 \gamma_{\mathrm{b}}$, where $\cos \theta=1$ is the cosine of the observation angle, assumed $\theta=0^{o}$, and $\beta_{\mathrm{b}}=v_{\mathrm{b}} / c$ is the velocity of the blob. Moreover, the $\gamma$-ray luminosity in the blob frame depends on the energy density of the synchrotron radiation and the Lorentz factor of the electrons,

$$
\dot{P}_{\mathrm{b}}^{\mathrm{IC}} \propto U_{\mathrm{syn}} \gamma_{\mathrm{e}}^{2} N_{\mathrm{e}},
$$

where $\gamma_{\mathrm{e}}$ is the Lorentz factor of the electrons that produce $\gamma$-rays (with characteristic energies for which a time delay has been reported), and $N_{\mathrm{e}}$ is the number of relativistic electrons inside the blob (assumed to be time-independent). The average energy density of synchrotron photons inside the blob is derived by estimating the synchrotron energy losses of the complete electron population inside the blob. That is, the energy losses of all electrons inside the blob multiplied by the average time that the synchrotron photons spend inside the blob, $\sim R_{\mathrm{b}} / c$, divided by the volume of the blob,

$$
U_{\text {syn }} \propto \frac{\dot{P}_{\text {syn }}}{V_{\mathrm{b}}} \frac{R_{\mathrm{b}}}{c},
$$

where

$$
\dot{P}_{\text {syn }} \propto B^{2} \gamma_{\mathrm{e}}^{2} N_{\mathrm{e}}
$$

are the synchrotron energy losses of electrons, $R_{\mathrm{b}}$ is the radius of the blob, and $V_{\mathrm{b}} \propto R_{\mathrm{b}}^{3}$ is the volume of the quasi-spherical blob. Provided that the energies of the electrons inside the blob are limited by their synchrotron energy losses, we compare their synchrotron energy losses with their energy gains from the acceleration mechanism, which scale as $\dot{P}_{\text {acc }} \propto B$ in the shock acceleration scenario. Then the maximum energy of the electrons is

$$
\gamma_{\mathrm{e}} \propto B^{-1 / 2} .
$$


In this case the synchrotron energy losses of the most energetic electrons are only linearly proportional to the magnetic field (Eq. 2.4). The blob is assumed to expand during its propagation along the jet proportionally to the distance traversed, i.e. its volume increases like $V_{\mathrm{b}} \propto X^{3}$. The $\gamma$-ray luminosity in the blob frame then depends on the distance from the base of the jet as $\dot{P}_{\mathrm{b}}^{\mathrm{IC}} \propto R_{\mathrm{b}}^{-2}$. Since the observed $\gamma$-ray luminosities $\left(\dot{P}_{\text {obs }}^{\mathrm{IC}}\right)$ in the considered energy range are approximately constant, the Doppler factor of the blob has to increase during the propagation of the blob along the jet like $D_{\mathrm{b}} \propto R_{\mathrm{b}}$ to keep the $\gamma$-ray luminosity approximately constant during the flare (cf. Eq. 2.1). Since the blob moves with a large Lorentz factor, the traversed distance is given approximately by $X \simeq c t \propto R_{\mathrm{b}}$, i.e., its Lorentz factor has to be approximately proportional to the time during which it travels inside the jet. Note that the time $t$ is measured in the stationary jet frame. Thus,

$$
\gamma_{\mathrm{b}} \simeq 0.5 D_{\mathrm{b}}=A t,
$$

where $A$ is a constant. We conclude that the observed time delay between different energies can be related to the acceleration phase of the blob inside the jet. In the next section, this conclusion is further investigated to constrain the blob acceleration scenario for the case of the recent observations of Mkn 501 [1].

\section{Energy-delayed $\gamma$-ray flare}

In our model, electrons are accelerated to maximum energies that change during the propagation of the blob due to a change of the local magnetic field strength in the jet. Thus, the $\gamma$-rays produced by these electrons also might have characteristic energies that are related to the energies of their parent electrons. The energy of a produced $\gamma$-ray photon can be estimated by

$$
E_{\gamma} \simeq m_{\mathrm{e}} \gamma_{\mathrm{e}} D_{\mathrm{b}} .
$$

This estimate is reasonable in the case of a relatively flat power-law spectrum of synchrotron photons (as observed during the Mkn 501 flares), i.e., when the inverse Compton scattering mainly occurs in the transition region between the Thomson and the Klein-Nishina regime. The time delay between the appearance of the flare at two different energies, $E_{\gamma}^{\min }$ and $E_{\gamma}^{\max }$, is due to the production of the corresponding $\gamma$-rays at different stages of the acceleration of the blob. While a photon with energy $E_{\gamma}^{\min }$ moves the distance $c \mathrm{~d} t$, the blob traverses a distance $\beta c \mathrm{~d} t$, where $\mathrm{d} t$ is the time measured in the jet frame, not in the blob frame. Therefore, the distance (in the jet frame) between two photons $E_{\gamma}^{\min }$ and $E_{\gamma}^{\max }$ is $\mathrm{d}(\Delta X)=c(1-\beta) \mathrm{d} t$. The distance between these photons corresponds to their time delay measured in the observer frame $\mathrm{d}(\Delta \tau)=\mathrm{d}(\Delta X) / c$. The total time delay in the case of a blob with changing velocity can be calculated as

$$
\Delta \tau=\int_{t_{\min }}^{t_{\max }}(1-\beta(t)) \mathrm{d} t \cong \int_{\gamma_{\mathrm{b}}^{\min }}^{\gamma_{\mathrm{m}}^{\max }} \frac{\mathrm{d} t}{2 \gamma_{\mathrm{b}}^{2}},
$$

where $1-\beta(t) \cong 1 / 2 \gamma_{\mathrm{b}}^{2}, t$ is the propagation time of the blob inside the jet, and $t_{\min }$ and $t_{\max }$ denote the moments at which the Lorentz factor of the blob is equal to $\gamma_{b}^{\min }$ and $\gamma_{b}^{\max }$, respectively. By applying the relation between the Lorentz factor of the blob and the travel time (Eq. 2.6), we arrive at a formula for the observational time delay between $\gamma$-rays of different energies

$$
\Delta \tau \cong \frac{1}{2 A} \int_{\gamma_{b}^{\min }}^{\gamma_{b}^{\max }} \frac{\mathrm{d} \gamma_{b}}{\gamma_{b}^{2}}=\frac{1}{2 A}\left(\frac{1}{\gamma_{b}^{\min }}-\frac{1}{\gamma_{b}^{\max }}\right) \approx \frac{1}{2 A \gamma_{b}^{\min }},
$$


provided that $\gamma_{\mathrm{b}}^{\max } \gg \gamma_{\mathrm{b}}^{\min }$. This simple relation for time delays in the flare observed at different photon energies holds true as long as these energies are directly linked to the energies of the parent electrons accelerated in the blob. It is obvious that the lower the energy ranges are chosen, the larger the observed time delay will be: For example, a time delay between $\gamma$-rays of $30 \mathrm{GeV}$ and $300 \mathrm{GeV}$ should be larger by an order of magnitude than a delay observed between $\gamma$-rays of $300 \mathrm{GeV}$ and $3 \mathrm{TeV}$. This clear prediction can be tested in the near future by simultaneous observations in the GeV energy range (with GLAST) and the TeV energy range (with MAGIC, H.E.S.S., and VERITAS).

During its acceleration from $\gamma_{b}^{\min }$ to $\gamma_{b}^{\max }$, the blob covers a distance

$$
\begin{aligned}
X_{\mathrm{acc}} & =c \int_{t_{\min }}^{t_{\max }} \beta(t) \mathrm{d} t \cong \frac{c}{A} \int_{\gamma_{\mathrm{b}}^{\min }}^{\gamma_{\mathrm{m}}^{\max }}\left(1-0.5 \gamma_{\mathrm{b}}^{-2}\right) \mathrm{d} \gamma_{\mathrm{b}} \\
& =\frac{c}{A}\left[\left(\gamma_{\mathrm{b}}^{\max }-\gamma_{\mathrm{b}}^{\min }\right)-\frac{1}{2}\left(\frac{1}{\gamma_{\mathrm{b}}^{\min }}-\frac{1}{\gamma_{\mathrm{b}}^{\max }}\right)\right] \\
& \approx \frac{c}{A}\left(\gamma_{\mathrm{b}}^{\max }-\gamma_{\mathrm{b}}^{\min }\right)
\end{aligned}
$$

in the jet. By using Eq. 3.3, we can express the distance $X_{\text {acc }}$ traveled by the blob by the measured time delay $\Delta \tau$ and the limiting values of the Lorentz factors of the blob, $X_{\mathrm{acc}}=c \Delta \tau\left(2 \gamma_{\mathrm{b}}^{\max } \gamma_{\mathrm{b}}^{\min }-1\right)$.

\section{Constraints on the Doppler factor of the blob}

Since a homogeneous SSC model is considered, we can constrain the Doppler factor of the blob in two ways based (a) on the known variability timescales of the synchrotron and $\gamma$-ray emission; and (b) on the escape condition of $\gamma$-rays from the synchrotron radiation inside the blob. The maximum energy of the observed synchrotron photons can be estimated by

$$
\varepsilon_{\mathrm{s}} \cong m_{\mathrm{e}} \frac{B}{B_{\mathrm{cr}}} \gamma_{\mathrm{e}}^{2} D_{\mathrm{b}} \cong m_{\mathrm{e}} \frac{B}{B_{\mathrm{cr}}}\left(\frac{E_{\gamma}^{\max }}{m_{\mathrm{e}}}\right)^{2} D_{\mathrm{b}}^{-1},
$$

where $B_{\mathrm{cr}}=4.4 \times 10^{13} \mathrm{G}$ and $\gamma_{\mathrm{e}}$ can be approximated by applying Eq. 3.1, $\gamma_{\mathrm{e}} \approx E_{\gamma}^{\max } /\left(m_{\mathrm{e}} D_{\mathrm{b}}\right)$. On the other hand, the observed emission variability timescale of the flare has to be at least equal to (or larger than) the synchrotron cooling time of the electrons to observe a synchrotron flare, i.e.,

$$
\tau_{\mathrm{var}} \geq \frac{\tau_{\mathrm{s}}}{D_{\mathrm{b}}}
$$

where $\tau_{\mathrm{s}}=3 m_{\mathrm{e}} \gamma_{\mathrm{e}} / C B^{2} \gamma_{\mathrm{e}}^{2}$, and $C$ is a constant describing the efficiency of synchrotron energy losses. By estimating the magnetic field strength from Eq. 4.1 and reversing Eq. 4.2, we obtain a lower limit on the Doppler factor of the blob,

$$
D_{\mathrm{b}}^{2} \geq \frac{E_{\gamma, \max }^{3}}{C B_{\mathrm{cr}}^{2} \tau_{\mathrm{var}} \varepsilon_{\mathrm{s}}^{2}}
$$

By considering the most extreme parameters ever observed for Mkn 501, i.e., $E_{\gamma, \max } \approx 20 \mathrm{TeV}[13$, 14 ], $\varepsilon_{\mathrm{s}} \approx 0.5 \mathrm{MeV}$ [15], and $\tau_{\mathrm{var}} \approx 200 \mathrm{~s}$ [1], we estimate the lower limit on the Doppler factor of the blob to be $D_{\mathrm{b}} \approx 10$, and the lower limit on the magnetic field strength inside the blob region 
to be $B \approx 0.3 \mathrm{G}$. Note that these values are consistent with estimates derived from more complete information on a flare in Mkn 501 (e.g., [8]) and with Doppler factors 25 - 50, which have been employed [1] for the modeling of the Mkn 501 multi-wavelength spectrum for the MAGIC 2005 observations. Note, however, that modeling the TeV $\gamma$-ray emission from PKS 2155-304, in which also very short timescale flares have been observed, seems to require Doppler factors on the order of $\sim 100$ [5].

We can also infer a lower limit on the Doppler factor of the blob by requiring the optical depth for $\gamma$-rays in the synchrotron radiation of the blob to be below unity. For another large flare observed from Mkn 501, the estimated the optical depth for $\gamma$-rays produced in the blob was estimated [8]. Based on eq. (6) of [8], using the above mentioned parameters for the 2005 July 9 flare, we estimate the blob Doppler factor to be $D_{\mathrm{b}} \geq 25$. Thus the blob has to move, at least during a part of its path inside the jet, with Lorentz factors $>10$. We now try to constrain the relation between the Lorentz factors of the blob during its acceleration phase by using the available information from observations in the context of the SSC model considered.

In the discussion above it was assumed that the maximum energies of the accelerated electrons are related to the magnetic field strength at the blob location (see Eq. 2.5), because the synchrotron process constitutes the dominant energy loss mechanism. But they are also related to the maximum energies of the $\gamma$-ray photons produced at the respective specific location of the blob inside the jet (see Eq. 3.1). In the considered model, the magnetic field in the blob drops like $B \propto X^{-1} \propto R_{\mathrm{b}}^{-1}$, with the Doppler factor of the blob $D_{\mathrm{b}} \propto R_{\mathrm{b}}$, and $\gamma_{\mathrm{e}} \propto B^{-1 / 2}$. Therefore, the energies of the observed $\gamma$-ray photons emitted at a specific location of the blob should be related to the Doppler factor of the blob by (see Eq. 3.1) $E_{\gamma} \propto D_{\mathrm{b}}^{3 / 2}$. The MAGIC collaboration reported the observation of a time delay between $\gamma$-rays at different energies $E_{\gamma}^{\min }$ and $E_{\gamma}^{\max }$. For the observed flare of Mkn 501 on 2005 July 9, these limiting values differ by approximately one order of magnitude. Therefore, the following relation should be fulfilled by the Doppler factors of the blob at the two characteristic blob locations in the jet, which correspond to the production of $\gamma$-rays with the energies $E_{\gamma}^{\min }$ and $E_{\gamma}^{\max }: D_{\mathrm{b}}^{\max } / D_{\mathrm{b}}^{\min } \approx \gamma_{\mathrm{b}}^{\max } / \gamma_{\mathrm{b}}^{\min } \approx 10^{2 / 3}$.

As an example, based on the above constraints, we consider a blob that is in its acceleration phase characterized by Lorentz factors $\gamma_{\mathrm{b}}^{\min }=10$ up to $\gamma_{\mathrm{b}}^{\max }=50$. For the reported time delay between the flare at different energies $(\Delta \tau=239 \mathrm{~s})$, Eq. 3.3 permits to estimate the value of the parameter $A \approx 1.7 \times 10^{-4} \mathrm{~s}^{-1}$. Eq. 3.4 yields the distance traveled by the blob during the acceleration phase between the two considered locations in the jet, $X_{\text {acc }} \approx 7 \times 10^{15} \mathrm{~cm}$. Note that this distance scale is $\sim 20$ times larger than the Schwarzschild radius of the $10^{9} \mathrm{M}_{\odot}$ black hole [16, 17] expected in the center of Mkn 501. Therefore, the blob acceleration region might well be located relatively close to the central engine. At such distances even the radiation field from the accretion disk around the black hole might prevent the escape of $\gamma$-ray photons (see, e.g., [18]). However, any conclusion on such effects is limited by the unknown radiation fields provided by the accretion disk and the broad line region in the case of BL Lac objects.

\section{Conclusions}

We have modified the classical homogeneous SSC model by allowing the blob to accelerate during its propagation along the inner part of the jet. The maximum energies of the electrons 
responsible for the $\gamma$-ray production are determined by the synchrotron process whose efficiency depends on the local strength of the magnetic field in the blob. Therefore, the maximum $\gamma$-ray energies are also related to the acceleration phase of the blob. In the resulting $\gamma$-ray flare, photons with lower energies are observed prior to those with higher energies. This modified SSC model can explain the recently observed arrival time difference of $\gamma$-rays belonging to different energies in a flare of Mkn 501. In our model, for the first time the MAGIC telescope was able to observe the acceleration phase of a blob in the jet of Mkn 501. Note that currently other models of the emission features at VHE $\gamma$-rays $[1,10]$ also provide consistent explanations.

Based on the measured time delay between the flares at different energies, we estimate the distance scale on which the acceleration of the blob occurred and predict that the time delay should increase inversely proportional to the energy of the $\gamma$-ray photons. The delay between the flare observed between $20 \mathrm{GeV}$ and $200 \mathrm{GeV}$ should be approximately an order of magnitude larger than the observed delay between $200 \mathrm{GeV}$ and $2 \mathrm{TeV}$. This clear prediction can be tested by simultaneous observations of strong flares at $\mathrm{GeV}$ and $\mathrm{TeV} \gamma$-ray energies using, e.g., the LAT instrument on board of GLAST (at GeV energies) and Cerenkov telescopes (at $\mathrm{TeV}$ energies).

\section{Acknowledgments}

RW acknowledges support from the DFG Excellence Cluster "Origin and Structure of the Universe". This research was supported by the Max Planck Society and the MNiI Grant 1P03D01028.

\section{References}

[1] J. Albert et al. (MAGIC Collab.) 2007, ApJ, 669, 862.

[2] A. P. Marscher \& W. K. Gear 1985, ApJ, 298, 11.

[3] L. Maraschi, G. Ghisellini, \& A. Celotti, 1992, ApJ, 397, L5.

[4] M. Giroletti et al. 2004, ApJ, 600, 127.

[5] F. Aharonian et al. (H.E.S.S. Collab.) 2007, ApJ, 664, L71.

[6] W. Benbow 2008, in these proceedings, POS (BLAZARS2 008 ) 012.

[7] S. Wagner 2008, in these proceedings, POS (BLAZARS2008) 021.

[8] W. Bednarek \& R. J. Protheroe 1999, MNRAS, 310, 577.

[9] M. C. Begelman, A. C. Fabian, \& M. J. Rees 2008, MNRAS, 384, L19.

[10] J. Albert et al. (MAGIC Collab.) 2007, preprint: arXiv:0708.2889 [astro-ph].

[11] P. G. Edwards \& B. G. Piner 2002, ApJ, 579, 67.

[12] W. Bednarek \& R. M. Wagner 2008, A\&A in press, preprint: arXiv:0804.0619 [astro-ph].

[13] A. Konopelko et al. (HEGRA Collab.) 1999, APh, 11, 135.

[14] F. Aharonian et al. (HEGRA Collab.) 2001, A\&A, 366, 62.

[15] M. Catanese et al. (Whipple Collab.) 1997, ApJ, 487, L143.

[16] R. Falomo, J. K. Kotilainen, \& A. Treves 2002, ApJ, 569, L33.

[17] A. J. Barth, L. C. Ho, \& W. L. W. Sargent 2003, ApJ, 583, 143.

[18] P. A. Becker \& M. Kafatos 1995, ApJ, 453, 83. 